\title{
A DESCRIPTION OF INCIDENCE RINGS OF GROUP AUTOMATA
}

\author{
A. V. KELAREV and D. S. PASSMAN
}

\begin{abstract}
Group automata occur in the Krohn-Rhodes Decomposition Theorem and have been extensively investigated in the literature. The incidence rings of group automata were introduced by the first author in analogy with group rings and incidence rings of graphs. The main theorem of the present paper gives a complete description of the structure of incidence rings of group automata in terms of matrix rings over group rings and their natural modules. As a consequence, when the ground ring is a field, we can use known group algebra results to determine when the incidence algebra is prime, semiprime, Artinian or semisimple. We also offer sufficient conditions for the algebra to be semiprimitive.
\end{abstract}

\section{Introduction}

Group automata can be briefly described as finite or infinite state machines where the input set can be identified with a group. The Krohn-Rhodes Decomposition Theorem tells us that an arbitrary finite state machine can be represented with a series-parallel connection of flip-flops and group automata, see [8, § 29] and [13]. The concepts of a group automaton and of a finite state automaton are also related to the notion of an inverse automaton and to that of a Cayley graph. See references $[\mathbf{1}],[\mathbf{2}],[\mathbf{3}],[\mathbf{9}],[\mathbf{1 0}]$, and $[\mathbf{1 5}]$ for recent results in these areas.

On the other hand, incidence algebras of graphs have also been actively investigated and many valuable results have been obtained, see [14]. A generalization of group algebras inspired by incidence rings of graphs was introduced recently in [7] using the concept of a finite group automaton, see Section 2. It is natural to expect that the incidence rings of group automata will play important roles in automata theory similar to the roles of group algebras in representation theory and in various applications in finite group theory.

The main result of this paper is Theorem 2.1. It completely describes the structure of all incidence rings of group automata in terms of matrix rings over group rings and their natural modules. Corollary 3.1 uses known group algebra results to determine when an incidence algebra is prime, semiprime, Artinian or

Key words and phrases. group automata; incidence rings; group rings.

The first author's research was supported by Discovery grant DP0449469 from the Australian Research Council. The second author's research was supported in part by NSA grant 144-PG40. 
semisimple, the latter being a generalization of the classical theorem of Maschke on group algebras of finite groups. Corollary 3.3 offers sufficient conditions for incidence algebras to be semiprimitive.

\section{The Main Result}

We use standard notation concerning automata, groups and rings, following references $[\mathbf{4}],[\mathbf{5}],[\mathbf{1 1}]$, and [13]. Recall that a group automaton is an algebraic system $\mathcal{A}=(X, G, \delta)$ where

(A1) $X$ is a nonempty set of states;

(A2) $G$ is a group, with identity element $1_{G}$, of input symbols;

(A3) $\delta: X \times G \rightarrow X$ given by $\delta(x, g)=x g$ is a transition function satisfying the equality

$$
x(g h)=(x g) h
$$

for all $x \in X, g, h \in G$.

In view of condition (1), $\delta$ is almost a permutation representation of $G$ on $X$. Indeed, all that is missing is the assumption that $x 1_{G}=x$ for all $x \in X$. Now it is easy to see that $X G=\{x g \mid x \in X, g \in G\}$ is the set of all elements of $X$ fixed by $1_{G}$. Thus, $\delta$ induces a permutation action of $G$ on $X G \subseteq X$, and clearly $\overline{\mathcal{A}}=(X G, G, \delta)$ is a subautomaton of $\mathcal{A}$. Of course, $X G$ is a disjoint union of $G$-orbits $\mathcal{O}$ and, for any $x \in X, x G$ is such an orbit. For convenience, we set $X^{\prime}=X \backslash X G$, and we let $\Omega_{G}$ denote the set of orbits of $G$ on $X G$.

If $x \in \mathcal{O}$, a $G$-orbit in $X G$, then we write $G_{x}$ for the stabilizer of $x$ in $G$, namely $G_{x}=\{g \in G \mid x g=x\}$. As is well known, $G_{x}$ is a subgroup of $G$ and the elements of $\mathcal{O}$ are in one-to-one correspondence with the right cosets of this subgroup. Thus $|\mathcal{O}|=\left|G: G_{x}\right|$. Furthermore, all such stabilizer subgroups of elements in the same $G$-orbit are conjugate in $G$ and hence they are isomorphic.

Let $F$ be a ring with 1 . Then the incidence ring of the automaton $\mathcal{A}=(X, G, \delta)$ over $F$ is the $\operatorname{ring} I_{\mathcal{A}}=I_{\mathcal{A}}(F)$ that is spanned as a free left $F$-module by the set $T_{\mathcal{A}}$ of all triples $\langle x, g, x g\rangle$, with $x \in X, g \in G$. Here multiplication is defined by the distributive law and the rules

$$
\begin{aligned}
\left\langle x_{1}, g_{1}, x_{1} g_{1}\right\rangle \cdot\left\langle x_{2}, g_{2}, x_{2} g_{2}\right\rangle & = \begin{cases}\left\langle x_{1}, g_{1} g_{2}, x_{1} g_{1} g_{2}\right\rangle & \text { if } x_{1} g_{1}=x_{2}, \\
0 & \text { otherwise }\end{cases} \\
\langle x, g, x g\rangle \cdot r & =r \cdot\langle x, g, x g\rangle,
\end{aligned}
$$

for all $x, x_{1}, x_{2} \in X, g, g_{1}, g_{2} \in G, r \in F$ (see [6] and [7] for more details).

For example, if $X=\{x\}$ is a singleton, then $x g=x$ for all $g \in G$, so $I_{\mathcal{A}}$ has as a free $F$-basis the elements $\langle x, g, x\rangle$ with $g \in G$ and with multiplication given by $\langle x, g, x\rangle \cdot\langle x, h, x\rangle=\langle x, g h, x\rangle$. Thus, in this case, $I_{\mathcal{A}}$ is naturally isomorphic to the group ring $F[G]$ via the map $\langle x, g, x\rangle \mapsto g$. Recall that if $G$ is a group, then the group ring $F[G]$ consists of all finite sums of the form $\sum_{g \in G} r_{g} g$, with $r_{g} \in F$, and with addition and multiplication defined by the rules

$$
\begin{gathered}
\sum_{g \in G} r_{g} g+\sum_{g \in G} r_{g}^{\prime} g=\sum_{g \in G}\left(r_{g}+r_{g}^{\prime}\right) g, \\
\left(\sum_{g \in G} r_{g} g\right) \cdot\left(\sum_{h \in G} r_{h}^{\prime} h\right)=\sum_{g, h \in G}\left(r_{g} r_{h}^{\prime}\right) g h .
\end{gathered}
$$


Now if $\mathcal{A}=(X, G, \delta)$ is arbitrary and if $T$ is a subset of the collection of triples $T_{\mathcal{A}}$, then we let $F T \subseteq I_{\mathcal{A}}$ denote the $F$-linear span of $T$. Thus, for example, it is clear that the incidence ring $I_{\overline{\mathcal{A}}}$ can be identified with $F\langle X G, G, X G\rangle$, where $\langle X G, G, X G\rangle=\{\langle x, g, x g\rangle \mid x \in X G, g \in G\}$. Similarly, if $\mathcal{O}$ is a $G$-orbit in $X G$, then $\mathcal{A}(\mathcal{O})=(\mathcal{O}, G, \delta)$ is also a subautomaton of $\mathcal{A}$ and $I_{\mathcal{A}(\mathcal{O})}$ can be identified with $F\langle\mathcal{O}, G, \mathcal{O}\rangle$, where $\langle\mathcal{O}, G, \mathcal{O}\rangle=\{\langle x, g, x g\rangle \mid x \in \mathcal{O}, g \in G\}$. Finally, we let $N_{\mathcal{A}}=F\left\langle X^{\prime}, G, X^{\prime} G\right\rangle$ be the $F$-linear submodule of $I_{\mathcal{A}}$ spanned by all triples $\langle x, g, x g\rangle$ with $x \in X^{\prime}=X \backslash X G, g \in G$.

In the following, if $S$ is a ring and $n$ is a cardinal number, possibly infinite, then we let $\mathbb{M}_{n}(S)$ denote the ring of all finite $n \times n$ matrices over $S$. In other words, each member of $\mathbb{M}_{n}(S)$ has only finitely many nonzero entries. Note that each row of $\mathbb{M}_{n}(S)$ is a right ideal and hence is naturally a right module for this ring. Furthermore, these modules are all isomorphic, and we denote any member of the isomorphism class by $\mathbb{R}_{n}(S)$. With this notation, our main result is

Theorem 2.1. Let $\mathcal{A}=(X, G, \delta)$ be a group automaton and let $F$ be a ring with 1. Then we have

(i) The F-incidence algebra $I_{\mathcal{A}}$ is a direct sum of $N_{\mathcal{A}}$ and $I_{\overline{\mathcal{A}}}$, where $\overline{\mathcal{A}}=$ $(X G, G, \delta)$. Here $I_{\overline{\mathcal{A}}}$ is an $F$-subalgebra of $I_{\mathcal{A}}$ and $N_{\mathcal{A}}$ is a 2-sided ideal of $I_{\mathcal{A}}$ with $I_{\mathcal{A}} \cdot N_{\mathcal{A}}=0$.

(ii) If $X G=\bigcup_{\mathcal{O} \in \Omega_{G}} \mathcal{O}$ is written as a disjoint union of $G$-orbits, then the ring $I_{\overline{\mathcal{A}}}$ is a direct sum of its 2-sided ideals $I_{\mathcal{A}(\mathcal{O})}$. Furthermore, if $x \in \mathcal{O}$ and if $H=G_{x}$ is the stabilizer subgroup of $x$ in $G$, then

$$
I_{\mathcal{A}(\mathcal{O})} \cong \mathbb{M}_{|\mathcal{O}|}(F[H]) \text {. }
$$

(iii) $N_{\mathcal{A}}$ is the direct sum of the $F$-linear spans $F\left\langle y, G, \mathcal{O}_{y}\right\rangle$, where $y \in X^{\prime}$ and $\mathcal{O}_{y}=y G$ is a $G$-orbit in $X G$. Each $F\left\langle y, G, \mathcal{O}_{y}\right\rangle$ is a 2-sided ideal of $I_{\mathcal{A}}$ with $F\left\langle y, G, \mathcal{O}_{y}\right\rangle \cdot I_{\mathcal{A}(\mathcal{O})}=0$ if $\mathcal{O} \neq \mathcal{O}_{y}$. Furthermore, when $\mathcal{O}=\mathcal{O}_{y}$ and when $I_{\mathcal{A}(\mathcal{O})}$ is written as the matrix ring $\mathbb{M}_{|\mathcal{O}|}(F[H])$, then $F\left\langle y, G, \mathcal{O}_{y}\right\rangle \cong$ $\mathbb{R}_{|\mathcal{O}|}(F[H])$ as a right $\mathbb{M}_{|\mathcal{O}|}(F[H])$-module.

Proof. (i) By definition, the $F$-basis $T_{\mathcal{A}}$ of $I_{\mathcal{A}}$ is a disjoint union of the basis of $N_{\mathcal{A}}$ and the basis of $I_{\overline{\mathcal{A}}}$. Hence $I_{\mathcal{A}}=N_{\mathcal{A}} \oplus I_{\overline{\mathcal{A}}}$ is a direct sum. Furthermore, since no element of $X^{\prime}=X \backslash X G$ can be the third element of a triple in $T_{\mathcal{A}}$, it follows that $I_{\mathcal{A}} \cdot N_{\mathcal{A}}=0$.

(ii) Let $X G=\bigcup_{\mathcal{O} \in \Omega_{G}} \mathcal{O}$ be written as a disjoint union of $G$-orbits. Since the $F$-basis $T_{\overline{\mathcal{A}}}$ of $I_{\overline{\mathcal{A}}}$ is a disjoint union of the $F$-bases $T_{\mathcal{A}(\mathcal{O})}$, it is clear that $I_{\overline{\mathcal{A}}}=\sum_{\mathcal{O} \in \Omega_{G}} I_{\mathcal{A}(\mathcal{O})}$ is a direct sum. Furthermore, if $\mathcal{O}$ and $\mathcal{O}^{\prime}$ are distinct orbits, then certainly $I_{\mathcal{A}(\mathcal{O})} \cdot I_{\mathcal{A}\left(\mathcal{O}^{\prime}\right)}=0$. Thus each $I_{\mathcal{A}(\mathcal{O})}$ is a 2 -sided ideal of $I_{\overline{\mathcal{A}}}$, and it remains to describe the multiplication in $I_{\mathcal{A}(\mathcal{O})}$.

To this end, fix $x \in \mathcal{O}$ and write $\mathcal{O}=\left\{x_{i} \mid i \in \mathcal{I}\right\}$ where $\mathcal{I}$ is an index set of size $|\mathcal{O}|$. Let $H=G_{x}$ be the stabilizer of $x$ in $G$ and, for each $i$, choose $g_{i} \in G$ with $x g_{i}=x_{i}$. Now the elements of $T_{\mathcal{A}(\mathcal{O})}$ are all of the form $\left\langle x_{i}, g, x_{j}\right\rangle$ with $x_{i} g=x_{j}$, and we note that the latter equation implies that $x g_{i} g g_{j}^{-1}=x_{i} g g_{j}^{-1}=x_{j} g_{j}^{-1}=x$. Thus $g_{i} g g_{j}^{-1} \in H=G_{x}$ and $g \in g_{i}^{-1} H g_{j}$. Conversely, it is easy to check that if $g \in g_{i}^{-1} H g_{j}$ then $x_{i} g=x_{j}$. With this observation, we can define an $F$-linear map $\theta: I_{\mathcal{A}(\mathcal{O})} \rightarrow \mathbb{M}_{|\mathcal{O}|}(F[H])$ by $\left\langle x_{i}, g, x_{j}\right\rangle \mapsto\left(g_{i} g g_{j}^{-1}\right) e_{i, j}$, where of course $g_{i} g g_{j}^{-1} \in H$ and $e_{i, j}$ is the matrix unit with 1 in the $(i, j)$ th entry and zeros elsewhere. 
Note that if $j \neq j^{\prime}$, then $\left\langle x_{i}, g, x_{j}\right\rangle \cdot\left\langle x_{j^{\prime}}, g^{\prime}, x_{k}\right\rangle=0$ and $e_{i, j} \cdot e_{j^{\prime}, k}=0$, so $\theta\left(\left\langle x_{i}, g, x_{j}\right\rangle\right) \cdot \theta\left(\left\langle x_{j^{\prime}}, g^{\prime}, x_{k}\right\rangle\right)=0$. On the other hand, if $j=j^{\prime}$ then

$$
\begin{aligned}
\theta\left(\left\langle x_{i}, g, x_{j}\right\rangle\right) \cdot \theta\left(\left\langle x_{j}, g^{\prime}, x_{k}\right\rangle\right) & =\left(g_{i} g g_{j}^{-1}\right) e_{i, j} \cdot\left(g_{j} g^{\prime} g_{k}^{-1}\right) e_{j, k} \\
& =g_{i} g g^{\prime} g_{k}^{-1} e_{i, k}=\theta\left(\left\langle x_{i}, g g^{\prime}, x_{k}\right\rangle\right) \\
& =\theta\left(\left\langle x_{i}, g, x_{j}\right\rangle \cdot\left\langle x_{j}, g^{\prime}, x_{k}\right\rangle\right)
\end{aligned}
$$

and $\theta$ is multiplicative. Thus $\theta$ defines an $F$-algebra homomorphism from $I_{\mathcal{A}(\mathcal{O})}$ to the ring $\mathbb{M}_{|\mathcal{O}|}(F[H])$ of finite matrices.

To see that $\theta$ is one-to-one and onto, we merely require a back map. Since the $F$-linear map $\theta^{\prime}: \mathbb{M}_{|\mathcal{O}|}(F[H]) \rightarrow I_{\mathcal{A}(\mathcal{O})}$ given by $\theta^{\prime}\left(h e_{i, j}\right)=\left\langle x_{i}, g_{i}^{-1} h g_{j}, x_{j}\right\rangle$, for all subscripts $i, j$ and all $h \in H$, is easily seen to be a well-defined inverse for $\theta$, the result follows. Note that, for any fixed subscript $i, \theta$ sends the $F$-linear span of the triples $\left\{\left\langle x_{i}, g, x_{i} g\right\rangle \mid g \in G\right\}$ onto the $i$ th row of $\mathbb{M}_{|\mathcal{O}|}(F[H])$.

(iii) As above, it is clear that $N_{\mathcal{A}}$ is the direct sum of the $F$-linear spans $F\left\langle y, G, \mathcal{O}_{y}\right\rangle$ with $y \in X^{\prime}=X \backslash X G$. Here $\mathcal{O}_{y}=y G$ is a $G$-orbit in $X G$ depending upon $y$, and $\left\langle y, G, \mathcal{O}_{y}\right\rangle$ is the set of triples of the form $\langle y, g, y g\rangle$ with $g \in G$. Now we know that $I_{\mathcal{A}} \cdot N_{\mathcal{A}}=0$, so the definition of multiplication implies immediately that $F\left\langle y, G, \mathcal{O}_{y}\right\rangle$ is a 2 -sided ideal of $I_{\mathcal{A}}$. It remains to determine the right $I_{\mathcal{A}}$-module structure of this ideal.

To this end, note that $y g=\left(y 1_{G}\right) g$ for all $g \in G$. Thus, the $F$-linear map $\phi: F\left\langle y, G, \mathcal{O}_{y}\right\rangle \rightarrow I_{\overline{\mathcal{A}}}$ given by $\langle y, g, y g\rangle \mapsto\left\langle y 1_{G}, g, y g\right\rangle$ is well defined and one-toone. Indeed, since these two triples have the same second and third entries, it is clear that $\phi$ defines a right $I_{\mathcal{A}}$-module homomorphism. Note that if $\mathcal{O}=\mathcal{O}_{y}$, then $y 1_{G} \in \mathcal{O}$, and hence the image of $\phi$ is contained in $I_{\mathcal{A}(\mathcal{O})}$. Now, in the notation of the proof of part (ii) above, $y 1_{G}=x_{i}$ for some $i \in \mathcal{I}$. Furthermore, as we have seen, the $F$-linear span of all triples of the form $\left\langle x_{i}, g, x_{i} g\right\rangle$, for all $g \in G$, maps onto the $i$ th row of $\mathbb{M}_{|\mathcal{O}|}(F[H])$ under the isomorphism $\theta: I_{\mathcal{A}(\mathcal{O})} \rightarrow \mathbb{M}_{|\mathcal{O}|}(F[H])$. Thus, as a right $I_{\mathcal{A}}$-module, $F\left\langle y, G, \mathcal{O}_{y}\right\rangle$ is isomorphic to $\mathbb{R}_{|\mathcal{O}|}(F[H])$ with $\mathcal{O}=\mathcal{O}_{y}$.

\section{Corollaries}

As we have seen, the structure of $I_{\mathcal{A}}$ is intimately related to the structure of finite matrix rings over certain group rings $F[H]$. In particular, this allows us to translate group ring results to incidence rings of group automaton. Since properties of $F[H]$ are better understood when $F$ is a field, we assume that this is the case in most of our results below.

We first consider conditions where the group algebra results are definitive. Recall that a ring $S$ is prime if the product of any two nonzero ideals is nonzero, and $S$ is semiprime if it has no nonzero nilpotent ideal. We say that $S$ is right or left Artinian if its right or left ideals satisfy the descending chain condition or equivalently the minimum condition. In the context of such rings, almost all radicals are equal. In particular, we say that $S$ is semisimple if it is semiprime and right or left Artinian.

Corollary 3.1. Let $\mathcal{A}=(X, G, \delta)$ be a group automaton and let $F$ be a field.

(i) $I_{\mathcal{A}}$ has an identity element if and only if $X=X G$ is finite.

(ii) $I_{\mathcal{A}}$ is right or left Artinian if and only if both $X$ and $G$ are finite.

(iii) If char $F=0$, then $I_{\mathcal{A}}$ is semiprime if and only if $X=X G$. 
(iv) If char $F=p>0$, then $I_{\mathcal{A}}$ is semiprime if and only if $X=X G$ and, for all $x \in X, G_{x}$ has no finite normal subgroup of order divisible by $p$.

(v) $I_{\mathcal{A}}$ is prime if and only if $X=X G$ is a single $G$-orbit and, for all $x \in X$, $G_{x}$ has no nonidentity finite normal subgroup.

(vi) If $\operatorname{char} F=0$, then $I_{\mathcal{A}}$ is semisimple if and only if $X=X G$ and $G$ are both finite.

(vii) If char $F=p>0$, then $I_{\mathcal{A}}$ is semisimple if and only if $X=X G$ and $G$ are both finite and, for all $x \in X, p$ does not divide the order of $G_{x}$.

Proof. Most of these facts are simple consequences of known group algebra results, so we just sketch some of the proofs. Of course, Theorem 1 and its notation will be used implicitly throughout the arguments.

(i) If $I_{\mathcal{A}}$ has an identity element, then certainly $N_{\mathcal{A}}=0$, so $X=X G$ and $I_{\mathcal{A}}=I_{\overline{\mathcal{A}}}$. Furthermore, $I_{\mathcal{A}}$ cannot be an infinite direct sum of ideals, so there are only finitely many $G$-orbits. Finally, the various rings $\mathbb{M}_{|\mathcal{O}|}(F[H])$ of finite matrices must each contain the identity matrix, so each such orbit size is finite. Consequently, $X=X G$ is finite, and the converse is clear.

(ii) If $I_{\mathcal{A}}$ is right or left Artinian, then so is $I_{\mathcal{A}} / N_{\mathcal{A}} \cong I_{\overline{\mathcal{A}}}$. Clearly, $I_{\overline{\mathcal{A}}}$ cannot involve an infinite direct sum of ideals, so there are only finitely many $G$-orbits in $X G$. Again, each matrix ring $\mathbb{M}_{|\mathcal{O}|}(F[H])$ cannot contain an infinite direct sum of rows or columns, so $|\mathcal{O}|$ is finite and hence $X G$ is finite. Next, note that each right or left ideal of $F[H]$ extends in a one-to-one manner to a right or left ideal of $\mathbb{M}_{|\mathcal{O}|}(F[H])$. Thus each $F[H]$ must be right or left Artinian, and hence each subgroup $H$ is finite by Theorem 10.1.1 of [11], a result of Connell. We conclude that $|G|=|H| \cdot|\mathcal{O}|$ is finite. Finally, $N_{\mathcal{A}}$ cannot be an infinite direct sum of the 2sided ideals $F\left\langle y, G, \mathcal{O}_{y}\right\rangle$ with $y \in X^{\prime}=X \backslash X G$. Thus $X^{\prime}$ is finite and consequently so is $X$. Conversely, if $X$ and $G$ are finite, then $I_{\mathcal{A}}$ is finite dimensional over $F$ and hence it is both right and left Artinian.

(iii)(iv) If $I_{\mathcal{A}}$ is semiprime, then certainly $N_{\mathcal{A}}=0$, so $X=X G$ and $\mathcal{A}=\overline{\mathcal{A}}$. Thus $I_{\mathcal{A}}$ is a direct sum of the various $I_{\mathcal{A}(\mathcal{O})}$, and $I_{\mathcal{A}}$ is semiprime if and only if each $I_{\mathcal{A}(\mathcal{O})} \cong \mathbb{M}_{|\mathcal{O}|}(F[H])$ is semiprime. Indeed, since any 2-sided ideal of the matrix ring $\mathbb{M}_{|\mathcal{O}|}(F[H])$ extends from an ideal of $F[H]$, we see that $\mathbb{M}_{|\mathcal{O}|}(F[H])$ is semiprime if and only if $F[H]$ is semiprime. Now, if $\operatorname{char} F=0$, then all $F[H]$ are semiprime by Theorem 4.2 .12 of [11] and consequently (iii) follows. On the other hand, if $\operatorname{char} F=p>0$, then by Theorem 4.2.13 of [11], a result of the second author, $F[H]$ is semiprime if and only if $H$ does not have a finite normal subgroup of order divisible by $p$. Since the groups $H$ that occur here are, up to isomorphism, all the stabilizer subgroups $G_{x}$ with $x \in X G$, we conclude that (iv) holds.

(v) If $I_{\mathcal{A}}$ is prime, then certainly $N_{\mathcal{A}}=0$, so $X=X G$ and $I_{\mathcal{A}}=I_{\overline{\mathcal{A}}}$. Furthermore, $I_{\mathcal{A}}$ cannot contain a nontrivial direct sum of 2-sided ideals, so $X G=\mathcal{O}$ must be a single $G$-orbit. This $I_{\mathcal{A}} \cong \mathbb{M}_{|\mathcal{O}|}(F[H])$ and, since every ideal of the matrix ring is extended from one of $F[H]$, the matrix ring is prime if and only if $F[H]$ is prime. It now follows from Theorem 4.2 .10 of $[\mathbf{1 1}]$, a result of Connell, that $I_{\mathcal{A}}$ is prime if and only if $H=G_{x}$ has no nonidentity finite normal subgroup.

(vi)(vii) Since a ring is semisimple if and only if it is Artinian and semiprime, these results follow from parts (ii), (iii) and (iv) above.

Note that parts (vi) and (vii) of the preceding corollary apply to finite group automaton and generalize the classical theorem of Maschke on group algebras, 
see Theorem 2.4.2 of [11]. We remark that there is an alternative proof of this generalization based on Theorem 6.1 of [6].

Next, we obtain a reduction of either the prime or Jacobson radical of $I_{\mathcal{A}}$ to the radicals of certain group rings. Here, we denote the prime radical of a ring $S$ by $\mathcal{P}(S)$ and the Jacobson radical of $S$ by $\mathcal{J}(S)$.

LEMma 3.2. Let $\mathcal{A}=(X, G, \delta)$ be an arbitrary group automaton and let $\mathcal{R}(S)$ denote either the prime or Jacobson radical of a ring $S$. Then

$$
\mathcal{R}\left(I_{\mathcal{A}}\right)=N_{\mathcal{A}} \oplus \mathcal{R}\left(I_{\overline{\mathcal{A}}}\right)=N_{\mathcal{A}} \oplus \sum_{\mathcal{O} \in \Omega_{G}} \mathcal{R}\left(I_{\mathcal{A}(\mathcal{O})}\right) .
$$

Furthermore, if $x \in \mathcal{O}$ and if $H=G_{x}$ is the stabilizer subgroup of $x$ in $G$, then $\mathcal{R}\left(I_{\mathcal{A}(\mathcal{O})}\right) \cong \mathbb{M}_{|\mathcal{O}|}(\mathcal{R}(F[H]))$.

Proof. We start with $\mathcal{R}=\mathcal{P}$, the prime radical. Recall that, for any ring $S$, $\mathcal{P}(S)$ is the intersection of all prime ideals of $S$ and hence also the intersection of all semiprime ideals of $S$. It follows that $\mathcal{P}\left(I_{\mathcal{A}}\right) \supseteq N_{\mathcal{A}}$ and that $\mathcal{P}\left(I_{\mathcal{A}}\right) / N_{\mathcal{A}}=$ $\mathcal{P}\left(I_{\mathcal{A}} / N_{\mathcal{A}}\right)=\mathcal{P}\left(I_{\overline{\mathcal{A}}}\right)$. Furthermore, since $I_{\overline{\mathcal{A}}}$ is the direct sum of the various $I_{\mathcal{A}(\mathcal{O})}$, it is clear that $\mathcal{P}\left(I_{\overline{\mathcal{A}}}\right)=\sum_{\mathcal{O} \in \Omega_{G}} \mathcal{P}\left(I_{\mathcal{A}(\mathcal{O})}\right)$. Finally, since every ideal of $\mathbb{M}_{|\mathcal{O}|}(F[H]) \cong I_{\mathcal{A}(\mathcal{O})}$ is extended from one of $F[H]$, it is easy to see that $\mathcal{P}\left(I_{\mathcal{A}(\mathcal{O})}\right) \cong$ $\mathcal{P}\left(\mathbb{M}_{|\mathcal{O}|}(F[H])\right)=\mathbb{M}_{|\mathcal{O}|}(\mathcal{P}(F[H]))$.

Next, for any ring $S$, its Jacobson radical $\mathcal{J}(S)$ is the intersection of all primitive 2-sided ideals of $S$, and it is also the largest quasi-regular ideal of the ring. Furthermore, if $e$ is an idempotent of $S$, then it is known that $\mathcal{J}(e S e)=e \mathcal{J}(S) e$. As a consequence of this and the fact that every 2-sided ideal of $\mathbb{M}_{n}(S)$ is extended from $S$, we have $\mathcal{J}\left(\mathbb{M}_{n}(S)\right)=\mathbb{M}_{n}(\mathcal{J}(S))$. With this, it follows as above that $\mathcal{J}\left(I_{\mathcal{A}}\right) \supseteq N_{\mathcal{A}}$ and that $\mathcal{J}\left(I_{\mathcal{A}}\right) / N_{\mathcal{A}}=\mathcal{J}\left(I_{\mathcal{A}} / N_{\mathcal{A}}\right)=\mathcal{J}\left(I_{\overline{\mathcal{A}}}\right)$. Moreover, since $I_{\overline{\mathcal{A}}}$ is the direct sum of the various $I_{\mathcal{A}(\mathcal{O})}$, the quasi-regular characterization of the Jacobson radical implies that $\mathcal{J}\left(I_{\overline{\mathcal{A}}}\right)=\sum_{\mathcal{O} \in \Omega_{G}} \mathcal{J}\left(I_{\mathcal{A}(\mathcal{O})}\right)$. Finally, the preceding matrix ring comments yield $\mathcal{J}\left(I_{\mathcal{A}(\mathcal{O})}\right) \cong \mathcal{J}\left(\mathbb{M}_{|\mathcal{O}|}(F[H])\right)=\mathbb{M}_{|\mathcal{O}|}(\mathcal{J}(F[H]))$, and the lemma is proved.

Note that the prime radical of group algebras is well understood due to the work of Dyment and Zalesskiu, see Theorem 8.4.16 of [11]. Consequently, we can use the preceding lemma to completely describe the prime radical of incidence algebras of group automaton. However, this description is quite technical group theoretically and therefore not appropriate for this short note. On the other hand, the group algebra semiprimitivity problem and the nature of the Jacobson radical of group algebras is far from settled. A fairly detailed survey of this material can be found in $[\mathbf{1 2}]$ and again the results are too technical to be considered here. Thus, we limit ourselves below to just one sufficient condition for semiprimitivity that is fairly easy to state. Recall that a possibly infinite group is said to be a $p^{\prime}$-group if it has no elements of finite prime order $p$.

Corollary 3.3. Let $\mathcal{A}=(X, G, \delta)$ be a group automaton with $X=X G$, and let $F$ be a field that is not algebraic over its prime subfield. If either char $F=0$ or char $F=p>0$ and each $G_{x}$, with $x \in X$, is a $p^{\prime}$-group, then the incidence algebra $I_{\mathcal{A}}(F)$ is semiprimitive.

Proof. This follows immediately from the preceding lemma with $\mathcal{R}=\mathcal{J}$. Indeed, $X=X G$ implies that $N_{\mathcal{A}}=0$, and our field and group assumptions 
combine with results of Amitsur, see Theorems 7.3.13 and 7.3.14 of [11], to conclude that $\mathcal{J}(F[H])=0$ for all $H=G_{x}$ with $x \in X$.

\section{Acknowledgement}

The first author is grateful to the second for organizing his sabbatical visit to the University of Wisconsin-Madison several years ago.

\section{References}

[1] J.-C. Birget, S. Margolis, J. Meakin and M. Sapir, Algorithmic Problems in Groups and Semigroups, Birkhäuser, Boston, 2000.

[2] J.-C. Birget, S. Margolis, J. Meakin and P. Weil, PSPACE-complete problems for subgroups of free groups and inverse finite automata, Theoret. Comput. Sci. 242 (2000) (1-2), 247-281.

[3] M. Delgado, S. Margolis and B. Steinberg, Combinatorial group theory, inverse monoids, automata, and global semigroup theory, Internat. J. Algebra Comput. 12 (2002) (1-2), 179211.

[4] J. M. Howie, Automata and Languages, Clarendon Press, Oxford, 1991.

[5] M. Ito, Algebraic Theory of Automata and Languages, World Scientific, River Edge, 2004.

[6] A. V. Kelarev, Ring Constructions and Applications, World Scientific, River Edge, 2002.

[7] A. V. Kelarev, On the structure of incidence rings of group automata, Internat. J. Algebra Comput. 14 (2004) (4), 505-511.

[8] R. Lidl and G. Pilz, Applied Abstract Algebra, Springer-Verlag, New York, 1998.

[9] S. W. Margolis and J. C. Meakin, E-unitary inverse monoids and the Cayley graph of a group presentation, J. Pure Appl. Algebra 58 (1989), 45-76.

[10] S. W. Margolis and J. C. Meakin, Free inverse monoids and graph immersions, Internat. J. Algebra Comput. 3 (1993) (1), 79-99.

[11] D. S. Passman, The Algebraic Structure of Group Rings, Wiley-Interscience, New York, 1977.

[12] D. S. Passman, Semiprimitivity of group algebras: past results and recent progress, Trends in ring theory (Miskolc, 1996), 127-157, CMS Conf. Proc., 22, Amer. Math. Soc., Providence, 1998.

[13] B. I. Plotkin, L. Ja. Greenglaz and A. A. Gvaramija, Algebraic Structures in Automata and Databases Theory, World Scientific, River Edge, 1992.

[14] E. Spiegel and C. J. O'Donnell, Incidence Algebras, Marcel Dekker, New York, 1997.

[15] B. Steinberg, Finite state automata: a geometric approach, Trans. Amer. Math. Soc. 353 (2001) (9), 3409-3464.

Graduate School of ITMS, University of Ballarat, P.O. Box 663, Ballarat, VicTORIA 3353, Australia

E-mail address: akelarev@staff.ballarat.edu.au

Mathematics Department, University of Wisconsin, 480 Lincoln Drive, Madison, WISCONSIN 53706, U.S.A.

E-mail address: passman@math.wisc.edu 